\title{
Author Correction: Telemedicine - maintaining quality during times of transition
}

\section{Sue Romanick-Schmiedl and Ganesh Raghu}

Nature Reviews Disease Primers (2020) https://doi.org/10.1038/s41572-020-0185-x Published online 01 June 2020.

The article's Author information has been updated to include the e-mail address of Sue Romanick-Schmiedl.

https://doi.org/10.1038/s41572-020-0199-4 I Published online: 07 July 2020

(๑) Springer Nature Limited 2020 